\title{
New Non-Orthogonal Transmission Schemes for Achieving Highly Efficient, Reliable, and Secure Multi-User Communications
}

\author{
JEHAD M. HAMAMREH ${ }^{1}$, MOHAMEDOU ABEWA ${ }^{1}$, LEMAYIAN JOEL PONCHA ${ }^{\mathbf{1}}$ \\ ${ }^{1}$ Department of Electrical and Electronics Engineering, Antalya Bilim University,Antalya, Turkey. \\ Authors are from WISLAB for Innovations in Wireless Communication \& Computation laboratory. \\ Corresponding author: J. M. Hamamreh (email: jehad.hamamreh@antalya.edu.tr, web: https://sites.google.com/view/wislab).
}

This work was supported in part by the Scientific and Technological Research Council of Turkey (TUBITAK), under project grant No. $119 \mathrm{E} 392$.

The Matlab simulation codes used to generate the results in this paper can be found at researcherstore.com

\begin{abstract}
Next-generation wireless communication paradigms demand properties such as high reliability, low power consumption, and enhanced security. Also, the ever-increasing demand for better wireless services has led to the continuous improvement and emergence of various wireless networks such as 5G and beyond networks. Beyond 5G communication systems (i.e., 6G) are envisioned to utilize technologies such as artificial intelligence, ultra-dense small cells, reconfigurable antennas, distributed networks, multi-band and full-duplex communications, as well as novel nonorthogonal multiple access methods. In this work, we first revisit and review the various current non-orthogonal multiple access (NOMA) techniques available in the literature and proposed by both academia and industry. Then, we discuss their strengths and weaknesses in different application areas. To address the limitations of the existing NOMA schemes, we develop and propose novel NOMA communication paradigms designed for achieving highly efficient, reliable, and secure multiuser communications using superimposed auxiliary signals and pre-coded matrices methods. The new proposed NOMA systems are motivated by the many limitations faced by current NOMA-based systems. For instance, power-domain NOMA is not included in release 17 of 3GPP as a work item. This is due to its performance degradation, resulting from successive interference cancellation (SIC) and channel estimation errors. The efficiency and novelty of the proposed models are presented via mathematical analysis and validated by Monte Carlo simulations.
\end{abstract}

INDEX TERMS 6G, 5G, Internet of Things, Low complexity, Non-Orthogonal Multiple Access (NOMA), Multi-user Superposition Transmission (MUST), 3rd Generation Partnership Project (3GPP), wireless communications, Physical Layer Security (PLS).

\section{INTRODUCTION}

$\mathbf{N}$ ew wireless communication networks have emerged due to the continued demand for better wireless services and the constant enhancement of wireless technology [1]. Future communication network technologies posses challenges such as energy efficiency (EE), since the entire network will be ultra-dense. The extremely high number of nodes may lead to a total power level above the acceptable limit. As such, 5G technology was envisioned to utilize technologies such as dense small cells and varied networks, device-to-device (D2D) communications, and multi-band and full-duplex communication. However, an unprecedented demand of new internet of things (IoT) applications such as extended reality (XR), telemedicine, flying cars, brain-computer interfaces, sensorless devices [2], and connected autonomous systems have emerged [3]. These new applications will disrupt the original goal of $5 \mathrm{G}$ of enabling short-packet and sensing-based Ultra Reliable Low Latency Communications (URLLC) and massive machine type communica- 
tion (mMTC) services. A wireless communication system designed to successfully siport the emerging IoT applications needs to have high reliability, low latency, and high data rates, for heterogeneous devices, for both uplink and downlink. Sixth generation (6G) wireless system is a disruptive wireless technology designed for these applications. 6G is envisioned to address challenges associated with the new applications such as the critical trade-off between rate, reliability, and latency as well as exploiting frequencies beyond sub- $6 \mathrm{GHz}$, therefore, transforming the wireless communication system into a self-sustaining intelligent and flexible paradigm with the ability to serve every IoT device according to its needs. In addition, novel multiple access techniques such as non-orthogonal multiple access (NOMA) technique will be on high demand to accommodate the rising number of connected devices.

Due to the scarcity of the available spectrum for wireless services, different generations of wireless systems have adopted and used multiple access techniques. Multiple access techniques are based on the idea of serving multiple users through the same frequency, time, code, and spatial resources simultaneously.

Moreover, all the previous wireless systems have used OMA (orthogonal multiple access) techniques such as frequency division multiple access (FDMA) in the first generation ( $1 \mathrm{G})$, time division multiple access (TDMA) in $2 \mathrm{G}$, code division multiple access (CDMA) in 3G, and orthogonal frequency division multiple access (OFDMA) in $4 \mathrm{G}$ [4].

Although OMA techniques provide a good system throughput performance with total robustness to interference due to the orthogonality between the user subcarriers, they have been proven unable to meet the requirements of the current $5 \mathrm{G}$ and upcoming $6 \mathrm{G}$ systems.

\section{A. TECHNICAL LITERATURE REVIEW}

To meet the demands of $6 \mathrm{G}$ networks, researchers have proposed NOMA as a strong candidate technique for meeting the harsh requirements of $5 \mathrm{G}$ and $6 \mathrm{G}$ networks. The main motivation behind the proposal of NOMA is its spectral efficiency and throughput, user fairness, low latency, massive connectivity and compatibility with future systems since it does not require significant modifications on the existing architecture

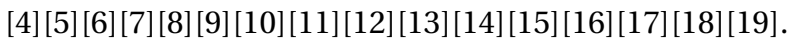

Depending on the domain used for multiplexing, conventional NOMA schemes are classified into powerdomain NOMA (PD-NOMA) and code-domain NOMA (CD-NOMA).

In PD-NOMA, users are assigned different power coefficients depending on their channel conditions. PDNOMA uses superposition coding at the transmitter side where multiple user's data signals are super-imposed and sent. At the receiver terminal, demodulation of the superimposed data signal is done through successive interference cancellation (SIC) until the desired user's signal is obtained.

On the other hand, CD-NOMA achieves multiplexing in the code-domain, similar to code-division multiple access (CDMA) or multi-carrier CDMA (MC-CDMA). However, unlike CDMA or MC-CDMA, code-domain NOMA uses low-density sequences or non-orthogonal sequences with low cross-correlation [20].

To improve different limitations of the basic CDNOMA and PD-NOMA, many interesting multiple access techniques have been developed including multi-user shared access (MUSA) [21], pattern division multiple access (PDMA) [22], resource spread multiple access (RSMA) [23], Welch-bound equality spread multiple access (WSMA) 24], interleave-grid multiple access (IGMA) [25], interleave division multiple access (IDMA) [26], and sparse-code multiple access (SCMA) [27].

All of the mentioned schemes are common in the use of the superposition principle and differ in the design of the signature of the user equipment which is based on spreading, coding, scrambling or interleaving.

Besides studies of NOMA schemes in the literature, many standardization activities have been attempted by different companies in the wireless industry. Examples of these standardization efforts are the proposed schemes in Release 14 of the 3rd generation partnership project (3GPP) as shown in Fig. 1 .

Particularly, the strongest candidate PD-NOMA, proposed under the name multi-user superposition transmission (MUST) which has been investigated in Release 14 till 16 of 3GPP has been rejected by the community and it is no more a work-item in Release 17 since it exhibits serious performance degradation resulting from treating interference as noise in the process of SIC and channel estimation errors compared to multiuser multiple-input-multiple-output (MU-MIMO) 66 28] 29].

\section{B. MOTIVATION}

The main limitation in NOMA is the user interference experienced by each user and security concerns. Also, proposing NOMA schemes that can work in power balanced scenarios is a point that is given much attention in the NOMA literature [30.

In general, the main drawbacks of conventional NOMA schemes are the following points:

- Conventional NOMA can work only for the cases where there is a significant path-loss channel difference between paired (or super-imposed) users. This necessitates having a user near to the base station (i.e., strong channel) and another user far from the base station (i.e., weak channel) [4] [5]. Consequently, this limits the applicability of NOMA in power-balanced scenarios, where two users may have the same path-loss as they are in the same region. 


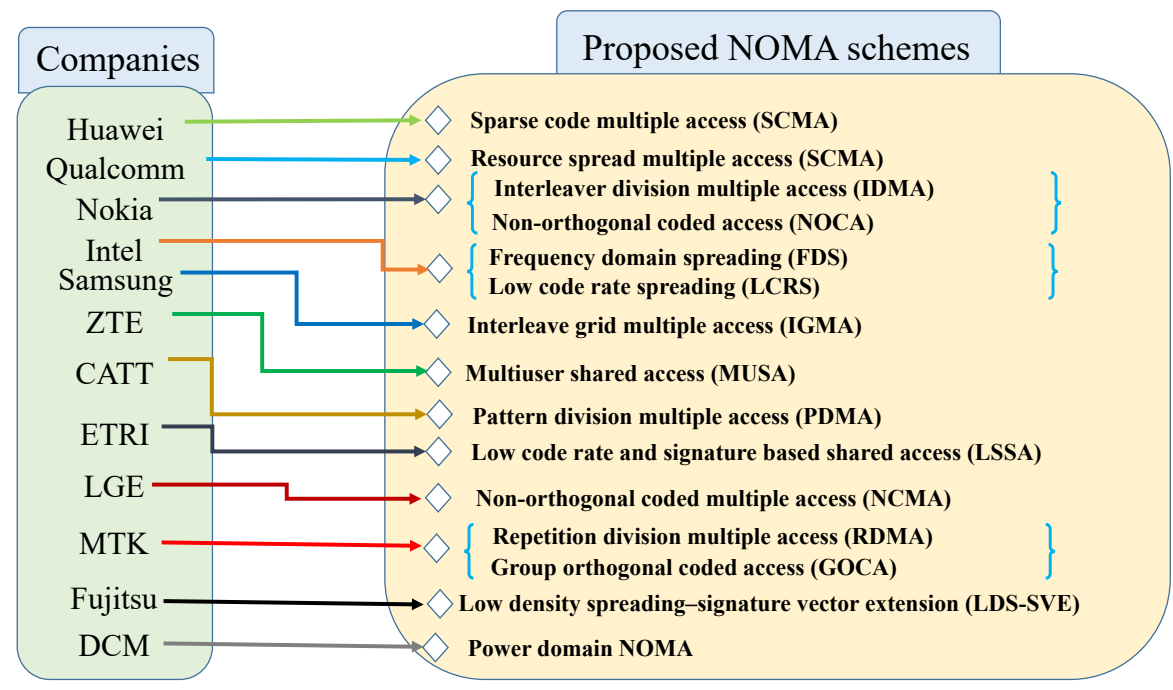

FIGURE 1. Examples of multiple access schemes proposed by different companies in Release 14 of $3 G P P$.

- Near users in conventional NOMA must use SIC to cancel the inter-user interference caused by the NOMA structure. This results in complicating the receiver structure which makes it inapplicable in the case of low complexity IoT devices that have minimal processing and power resources.

- The user with a good channel always decodes the message of the other user first before decoding their message. Hence, conventional NOMA has limited internal security.

- The far user always treats the interference of near user as noise, where it decodes the received signal directly as if it were its own pure signal, which causes degradation in its reliability performance.

\section{OUR CONTRIBUTIONS}

To address these challenges, four novel non-orthogonal communication designs are proposed in this paper. In the proposed paradigms, signal pre-coding, as well as auxiliary signal superposition are utilized. The main contribution points of these designs are summarized as follows:

- Canceling the interference by means of signal precoding and auxiliary signal superposition, thus, removing the need for SIC at the receivers which results in low system complexity and low power consumption.

- Regardless of their distances from the BS, any two users can be superimposed. This solves the problem of conventional NOMA relating to the application in power-balanced scenarios.

- Achieving the same reliable performance as an OMA system while having the enhancements of NOMA.

- Enhancing internal and external security by utilizing PLS.

The new NOMA techniques are designed such that signal transmission complexity such as equalization, interference cancellation and others are completely moved to the base station.

The remainder of this work is organized as follows: The proposed design architectures are explained in details in section II. Section III discusses performance analysis by investigating different performance metrics . Finally, section IV presents the conclusion.

\section{PROPOSED DESIGNS}

In this section, the proposed NOMA inspired techniques will be discussed. There are four distinct NOMA inspired methods presented in this work modeled around the following two major methods:

- NOMA with signal precoding.

- NOMA with auxiliary signal superposition.

Under each technique, two designs are developed by exploring time diversity and spatial transmit diversity of the transmitted signal.

In the following subsections $\mathbf{H}_{\mathbf{i}, \mathbf{j}}$ is used to indicate the frequency response of the channel from antenna $\mathrm{i}$ to user $\mathrm{j}$. This communication techniques are applicable for any number of users, however, this work employs two users scenario for simplicity purposes, hence, the notations $\mathbf{H}_{11}, \mathbf{H}_{12}$ represents the frequency response of the channel from antenna-1 to user $_{1}$ and from antenna2 to user $_{2}$ respectively. $\mathbf{H}_{21}$ and $\mathbf{H}_{22}$ denote the channel frequency response between antenna-2 and user $_{1}$ and antenna-2 and user $_{2}$, respectively. Similarly, $\mathbf{H}_{31}$ and $\mathbf{H}_{32}$ is the frequency response of the channel between antenna- 1 and the eavesdropper, and antenna- 2 and the eavesdropper respectively.

\section{A. NON-ORTHOGONAL SECURE TRANSMISSION DESIGNS THROUGH SIGNAL PRE-CODING}


1) NOMA with signal pre-coding using time diversity $\left(C T_{1}\right]^{1}$

The proposed system consists of a multi-carrier OFDM system employing dual antennas trying to communicate with two single-antenna legitimate users, in the presence of one single-antenna passive eavesdropper as shown in Fig. 2 Signal transmission happen in two rounds, during each transmission round, only one antenna is made active.

From the described model, consider an OFDM system with $N$ modulated symbols in each OFDM block for all users. The frequency response of each OFDM symbol for user $_{1}$ can be shown as $\mathbf{x}_{\mathbf{1}}=\left[x_{0}, x_{1}, \ldots . ., x_{N-1}\right]$ where $\mathbf{x}_{1} \in C^{[N \times 1]}$. Also, the frequency response vector of user $_{2}$ can be represented as $\mathbf{x}_{2}=\left[x_{0}, x_{1}, \ldots \ldots, x_{N-1}\right]$, where $\mathbf{x}_{\mathbf{2}} \in C^{[N \times 1]}$. The basic idea behind this proposed paradigm is to first multiply each user's signal with a specially designed channel dependent pre-coder matrix. Then superimpose the pre-coded signals and send it in the first and the second transmission rounds. The superimposed signal is sent such that each user gets their own intended signal just by demodulating the received signal and without any extra complex processing. On the other hand, the eavesdropper will not be able to decode the information intended for user $_{1}$ and user $_{2}$ due to the channel dependent pre-coder matrices. Moreover, the matrices will also ensure that there is no eavesdropping between user $_{1}$ and user $_{2}$, otherwise known as internal eavesdropping.

Based on the design of the paradigm discussed above, the pre-coded superimposed user signal transmitted during the first round on antenna-1 is given as:

$$
\mathbf{u}_{1}=\mathbf{M}_{1 \mathrm{a}} \mathbf{x}_{1}+\mathbf{M}_{2 \mathrm{a}} \mathbf{x}_{2} .
$$

Similarly, the pre-coded superimposed user signal transmitted during the second round on antenna-2 is given as:

$$
\mathbf{u}_{2}=\mathbf{M}_{1 \mathrm{~b}} \mathbf{x}_{\mathbf{1}}+\mathbf{M}_{\mathbf{2 b}} \mathbf{x}_{\mathbf{2}}
$$

where $\mathbf{x}_{1}$ and $\mathbf{x}_{2}$ are user $_{1}$ and user $_{2}$ respective signals in frequency domain, while $\mathbf{M}_{\mathbf{1 a}}, \mathbf{M}_{\mathbf{2}}, \mathbf{M}_{\mathbf{1 b}}$, and $\mathbf{M}_{\mathbf{2 b}}$ are the legitimate user's channel dependent pre-coder matrices.

The received signal at user $_{1}$ is the combined signal transmitted during the first and the second round through $\mathbf{H}_{11}$ and $\mathbf{H}_{12}$, depicted as:

$$
\hat{\mathbf{y}}_{1}=\mathbf{H}_{11}^{\mathrm{H}} \mathbf{y}_{11}+\mathbf{H}_{12}^{\mathrm{H}} \mathbf{y}_{12},
$$

where $\mathbf{y}_{11}$ is the received signal at user $_{1}$ during transmission round 1 , and $\mathbf{y}_{12}$ is the received signal at $u s e r_{1}$ during transmission round 2. Expanding (3) we get:

$$
\hat{\mathbf{y}}_{1}=\left|\mathrm{H}_{11}\right|^{2} \mathbf{u}_{1}+\mathbf{H}_{11}^{\mathrm{H}} \mathbf{z}_{11}+\left|\mathrm{H}_{12}\right|^{2} \mathbf{u}_{2}+\mathbf{H}_{11}^{\mathrm{H}} \mathbf{z}_{12},
$$

\footnotetext{
${ }^{1}$ The four proposed non-orthogonal Communication Technique (CT) designs will be refereed to as CT 1 to 4 .
}

$$
\begin{aligned}
\hat{\mathbf{y}}_{\mathbf{1}}= & \left(\left|\mathbf{H}_{11}\right|^{2} \mathbf{M}_{1 \mathbf{a}}+\left|\mathbf{H}_{12}\right|^{2} \mathbf{M}_{1 \mathbf{b}}\right) \mathbf{x}_{1} \\
& +\left(\left|\mathbf{H}_{11}\right|^{2} \mathbf{M}_{2 \mathbf{a}}+\left|\mathbf{H}_{12}\right|^{2} \mathbf{M}_{2 \mathbf{b}}\right) \mathbf{x}_{\mathbf{2}}+\mathbf{H}_{11}^{\mathrm{H}} \mathbf{z}_{11}+\mathbf{H}_{11}^{\mathbf{H}} \mathbf{z}_{12},
\end{aligned}
$$

where $\mathbf{z}_{11}$ and $\mathbf{z}_{12}$ are the noise terms for user 1 during round 1 and round 2 , respectively.

The received signal at user $_{2}$ is also the combined transmitted signal during round 1 and round 2 through $\mathbf{H}_{21}$ and $\mathbf{H}_{22}$, calculated as:

$$
\hat{\mathbf{y}}_{2}=\mathbf{H}_{21}^{\mathrm{H}} \mathbf{y}_{21}+\mathbf{H}_{22}^{\mathrm{H}} \mathbf{y}_{22} \text {, }
$$

where $\mathbf{y}_{21}$ is the received signal at user $_{2}$ during round 1 through antenna- 1 and $\mathbf{y}_{22}$ is the received signals at user $_{2}$ during round 2 through antenna-2. After expanding [6] and substituting $\mathbf{u}_{\mathbf{1}}$ and $\mathbf{u}_{2}$, we get:

$$
\begin{gathered}
\hat{\mathbf{y}}_{2}=\left|\mathbf{H}_{21}\right|^{2} \mathbf{u}_{1}+\mathbf{H}_{21}^{\mathrm{H}} \mathbf{z}_{21}+\left|\mathbf{H}_{22}\right|^{2} \mathbf{u}_{2}+\mathbf{H}_{22}^{\mathrm{H}} \mathbf{z}_{22}, \\
\hat{\mathbf{y}}_{2}=\left(\left|\mathbf{H}_{21}\right|^{2} \mathbf{M}_{1 \mathbf{a}}+\left|\mathbf{H}_{22}\right|^{2} \mathbf{M}_{1 \mathbf{b}}\right) \mathbf{x}_{1} \\
+\left(\left|\mathbf{H}_{21}\right|^{2} \mathbf{M}_{2 \mathbf{a}}+\left|\mathbf{H}_{22}\right|^{2} \mathbf{M}_{2 \mathbf{b}}\right) \mathbf{x}_{2}+\mathbf{H}_{21}^{\mathrm{H}} \mathbf{z}_{21}+\mathbf{H}_{22}^{\mathrm{H}} \mathbf{z}_{22} .
\end{gathered}
$$

Using (5) and (8) we now model the pre-coder matrices as follows. The first term of $[5$ is the desired term for user $_{1}$, hence $\left(\left|\mathbf{H}_{11}\right|^{2} \mathbf{M}_{1 \mathbf{a}}+\left|\mathbf{H}_{12}\right|^{2} \mathbf{M}_{1 \mathbf{b}}\right)=\mathbf{I}$. Also, to cancel the interference of user $_{1}$ to user $_{2}$, the first term of 8 is set equal to 0, that is $\left(\left|\mathbf{H}_{21}\right|^{2} \mathbf{M}_{\mathbf{1 a}}+\left|\mathbf{H}_{22}\right|^{2} \mathbf{M}_{1 \mathbf{b}}\right)=\mathbf{0}$. Solving this two equations together, we find $\mathbf{M}_{\mathbf{l a}}$ and $\mathbf{M}_{\mathbf{l b}}$ as follows:

$$
\begin{array}{r}
M_{1 \mathrm{a}}=\left|\mathrm{H}_{22}\right|^{2}\left(\left|\mathrm{H}_{22}\right|^{2}\left|\mathrm{H}_{11}\right|^{2}-\left|\mathrm{H}_{21}\right|^{2}\left|\mathrm{H}_{12}\right|^{2}\right)^{-1}, \\
\mathbf{M}_{1 \mathrm{~b}}=-\left|\mathrm{H}_{21}\right|^{2}\left(\left|\mathrm{H}_{22}\right|^{2}\left|\mathrm{H}_{11}\right|^{2}-\left|\mathrm{H}_{21}\right|^{2}\left|\mathrm{H}_{12}\right|^{2}\right)^{-1} .
\end{array}
$$

To model $\mathbf{M}_{\mathbf{2 a}}$ and $\mathbf{M}_{\mathbf{2 b}}$ we follow similar steps. To remove the effect of the channel on user $_{2}$, the second term of 8 is set to identity such that $\left(\left|\mathbf{H}_{21}\right|^{2} \mathbf{M}_{2 \mathbf{a}}+\right.$ $\left.\left|\mathbf{H}_{22}\right|^{2} \mathbf{M}_{2 \mathbf{b}}\right)=\mathbf{I}$, while the second term of 5 is set to 0 so as to remove the interference caused by user $_{2}$ on user $_{1}$, that is $\left(\left|\mathbf{H}_{11}\right|^{2} \mathbf{M}_{2 \mathbf{a}}+\left|\mathbf{H}_{\mathbf{1 2}}\right|^{2} \mathbf{M}_{\mathbf{2 b}}\right)=\mathbf{0}$. Using this two equations we find $\mathbf{M}_{\mathbf{2 a}}$ and $\mathbf{M}_{\mathbf{2 b}}$ as follows:

$$
\begin{gathered}
\mathbf{M}_{2 \mathrm{a}}=\left|\mathrm{H}_{12}\right|^{2}\left(\left|\mathrm{H}_{12}\right|^{2}\left|\mathrm{H}_{21}\right|^{2}-\left|\mathrm{H}_{11}\right|^{2}\left|\mathrm{H}_{22}\right|^{2}\right)^{-1}, \\
\mathbf{M}_{2 \mathrm{~b}}=-\left|\mathrm{H}_{11}\right|^{2}\left(\left|\mathrm{H}_{12}\right|^{2}\left|\mathrm{H}_{21}\right|^{2}-\left|\mathrm{H}_{11}\right|^{2}\left|\mathrm{H}_{22}\right|^{2}\right)^{-1} .
\end{gathered}
$$

The specially designed pre-coder matrices will make sure that user $_{1}$ and user $_{2}$ receive their intended signals without performing any extra processing at the receiver. Moreover, the signals will be secure from internal and external eavesdropping.

\section{2) NOMA with signal pre-coding using spatial transmit diversity $\left(C T_{2}\right.$}

In this design, signal pre-coding is used with spatial transmit diversity of the base station to provide a reliable and completely secured communication. Similar to the aforementioned method, this design does not use SIC at the receiver and relies solely on the design of the precoding matrices that cancel both the internal and external eavesdropping. 


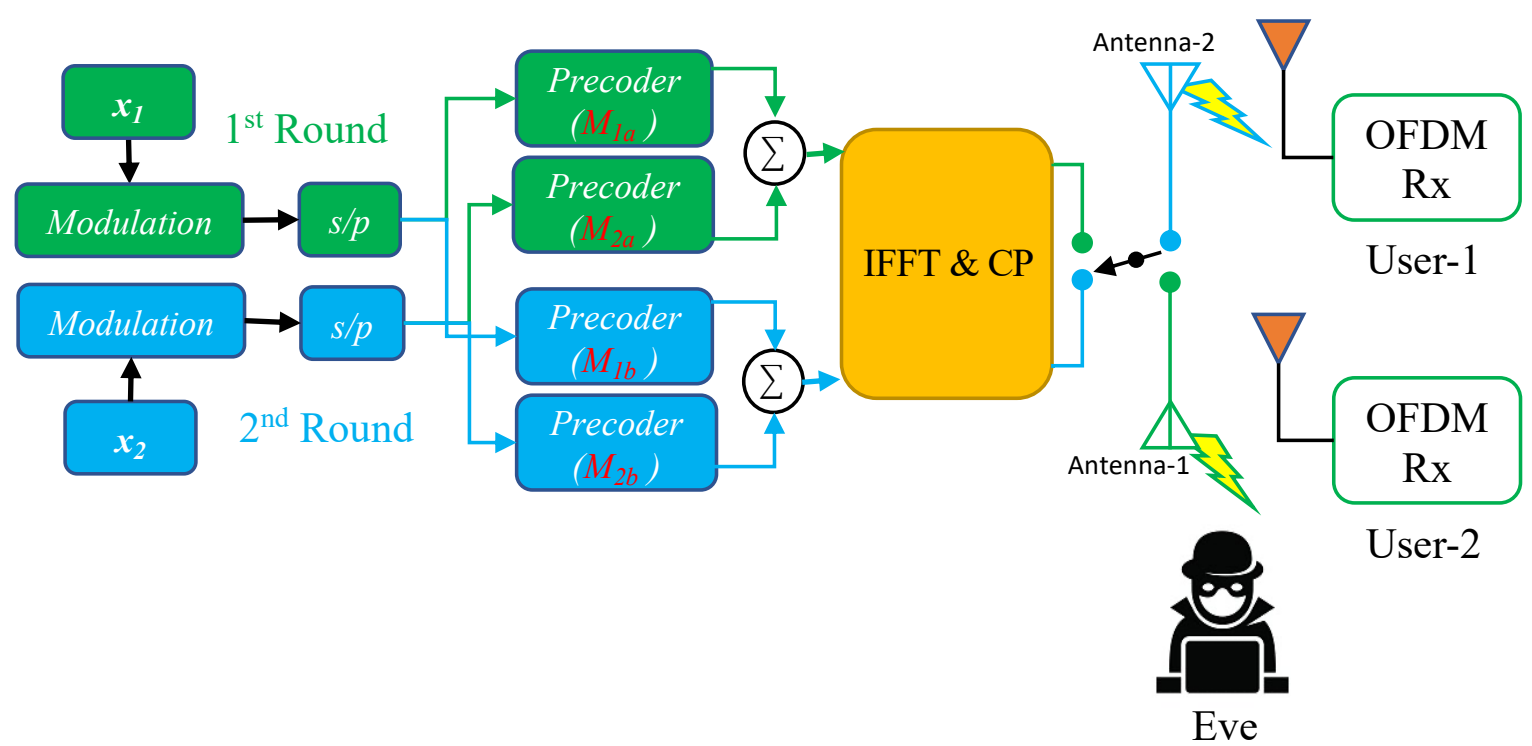

FIGURE 2. NOMA with signal pre-coding using time diversity (i.e., dual transmission enabled by MISO) $\left(C T_{1}\right)$

The basic detailed diagram of the proposed algorithm is presented in Fig. 3. In the considered system model, it is assumed that there is a NOMA-OFDM transmitter that wants to communicate with the two users securely, where users can only get their own data and an external eavesdropper cannot access any of the two users' data.

As shown in Fig. 3 the design is based on OFDM where each OFDM block consists of $N$ number of QAM modulated symbols. The frequency domain representation of user $_{1}$ (i.e $\mathbf{x}_{1}$ ) and user $_{2}$ (i.e $\mathbf{x}_{2}$ ) can be written as: $\mathbf{x}_{1}=\left[x_{1}[0], x_{1}[1],:::, x_{1}[N-1]\right], \mathbf{x}_{2}=\left[x_{2}[0], x_{2}[1],:::, x_{2}[N-1]\right]$

Afterwards, $\mathbf{x}_{1}$ and $\mathbf{x}_{2}$ are converted from serial to parallel, then multiplied by pre-coded matrices, added (superimposed) to each other, the IFFT of the superimposed signal is then taken and the cyclic prefix is added and finally the superimposed signal is simultaneously transmitted from the two antennas.

The transmitted signal from antenna-1, after precoding with respect to $u_{s e r}$ and $u s e r_{2}$, is given as follows:

$$
\mathbf{u}_{1}=\mathbf{M}_{1 \mathrm{a}} \mathbf{x}_{1}+\mathbf{M}_{2 \mathrm{a}} \mathbf{x}_{2}
$$

Similarly, the transmitted signal from antenna-2, after pre-coding, is given as:

$$
\mathbf{u}_{2}=\mathbf{M}_{1 \mathbf{b}} \mathbf{x}_{1}+\mathbf{M}_{2 b} \mathbf{x}_{2}
$$

where $\mathbf{M}_{\mathbf{1 a}}, \mathbf{M}_{\mathbf{2 a}}, \mathbf{M}_{\mathbf{1 b}}, \mathbf{M}_{\mathbf{2 b}}$ are pre-coding matrices. The values of these matrices are chosen in such a way to cancel the inter-user interference both internally and externally and to cancel the channel effect at each legitimate user. The pre-coded signal simultaneously sent from the transmitters reach user $_{1}$ at the same time, hence, the received signal at $u_{\text {ser }}{ }_{1}$ is calculated as:

$$
\hat{\mathbf{y}}_{1}=\mathbf{y}_{11}+\mathbf{y}_{12} \text {, }
$$

where $\mathrm{y}_{11}$ and $\mathrm{y}_{12}$ are the received signals at $u$ ser 1 through antenna-1 and antenna-2, respectively. Substituting $\mathbf{y}_{11}$ and $\mathbf{y}_{12}$ in (15), the combined signal can be presented as follows:

$$
\hat{\mathbf{y}}_{1}=\mathrm{H}_{11} \mathbf{u}_{1}+\mathrm{z}_{11}+\mathrm{H}_{12} \mathbf{u}_{2}+\mathrm{z}_{12}
$$

where $z_{11}$ and $z_{12}$ are the noise terms for user $_{1}$ and user $r_{2}$. Substituting the values of $\mathbf{u}_{1}$ and $\mathbf{u}_{2}$ from 13 and 14] and simplifying, we get:

$$
\begin{aligned}
\hat{\mathbf{y}} 1= & H_{11}\left(M_{1 a} x_{1}+M_{2 a} x_{2}\right)+z_{11} \\
& +H_{12}\left(M_{1 b} x_{1}+M_{2 b} x_{2}\right)+z_{12} \\
\hat{y}_{1}= & \left(H_{11} M_{1 a}+H_{12} M_{1 b}\right) \mathbf{x}_{1}+\left(H_{11} M_{2 a}\right. \\
& \left.+H_{12} M_{2 b}\right) \mathbf{x}_{2}+z_{11}+z_{12} .
\end{aligned}
$$

Similar to user $_{1}$, the combined received signal from antenna-1 and antenna-2 for the case of user $_{2}$ can be written as:

$$
\hat{\mathbf{y}}_{2}=\mathbf{y}_{21}+\mathbf{y}_{22} \text {, }
$$

where $\mathbf{y}_{21}=\mathbf{H}_{21} \mathbf{u}_{1}+\mathbf{z}_{21}$ and $\mathbf{y}_{22}=\mathbf{H}_{22} \mathbf{u}_{2}+\mathbf{z}_{22}$ are the received signals at $u_{\text {ser }}$ through antenna- 1 and antenna2, respectively. $\mathbf{z}_{21}$ and $\mathbf{z}_{22}$ are the noise terms for $u_{s e r}$ related to antenna-1 and antenna-2, respectively.

Substituting the values of $\mathbf{u}_{\mathbf{1}}$ and $\mathbf{u}_{\mathbf{2}}$ from 13 and 14 and simplifying, we get:

$$
\begin{aligned}
\hat{\mathbf{y}}_{2}= & \mathbf{H}_{21}\left(\mathbf{M}_{1 \mathbf{a}} \mathbf{x}_{1}+\mathbf{M}_{2 \mathrm{a}} \mathbf{x}_{2}\right)+\mathbf{z}_{21} \\
& +\mathbf{H}_{22}\left(\mathbf{M}_{1 \mathbf{b}} \mathbf{x}_{1}+\mathbf{M}_{2 \mathrm{~b}} \mathbf{x}_{2}\right)+\mathbf{z}_{22}, \\
\hat{\mathbf{y}}_{2}=( & \left.\mathrm{H}_{21} \mathbf{M}_{1 \mathbf{a}}+\mathbf{H}_{22} \mathbf{M}_{1 \mathbf{b}}\right) \mathbf{x}_{1}+\left(\mathbf{H}_{21} \mathbf{M}_{2 \mathrm{a}}\right. \\
& \left.+\mathbf{H}_{22} \mathbf{M}_{2 \mathbf{b}}\right) \mathbf{x}_{2}+\mathbf{z}_{21}+\mathbf{z}_{22}
\end{aligned}
$$




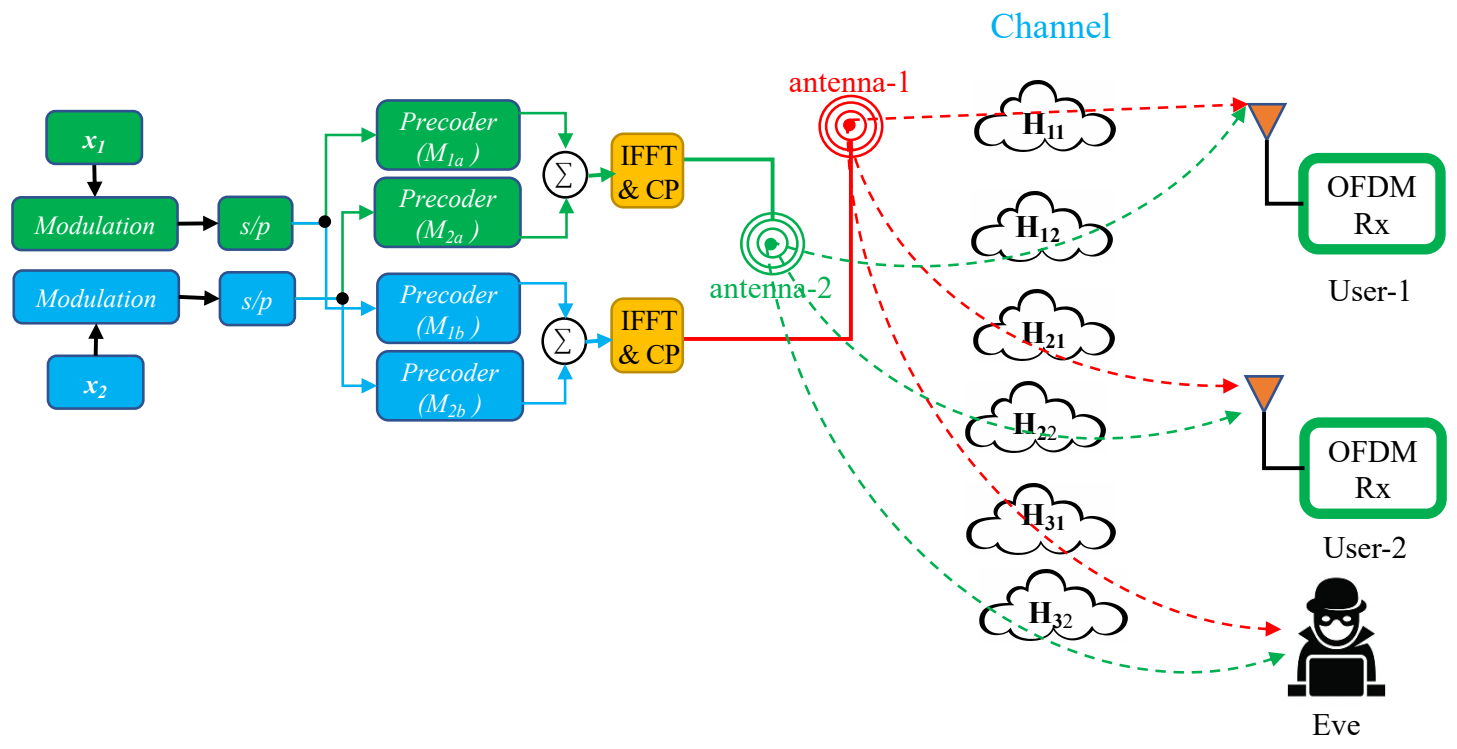

FIGURE 3. NOMA with signal pre-coding using spatial transmit diversity $\left(C T_{2}\right)$

The pre-coded matrices will ensure that the signal is decoded at each intended user without extra processing and that the signals are secure from internal and external eavesdropping.

Through a similar calculation as detailed in the design of the matrices in the section above, the precoded matrices in this case can be found by solving the system of equations composed of the desired and undesired term for each user. The desired term is set to identity and the undesired term is suppressed by setting it to the null matrix. The values resulting from the solution of this system of equations are as follows for antenna-1:

$$
\begin{gathered}
M_{1 a}=H_{22}\left(H_{22} H_{11}-H_{21} H_{12}\right)^{-1}, \\
M_{1 b}=-H_{21}\left(H_{22} H_{11}-H_{21} H_{12}\right)^{-1} .
\end{gathered}
$$

and for antenna-2:

$$
\begin{gathered}
M_{2 a}=H_{12}\left(H_{12} H_{21}-H_{11} H_{22}\right)^{-1}, \\
M_{2 b}=-H_{11}\left(H_{12} H_{21}-H_{11} H_{22}\right)^{-1} .
\end{gathered}
$$

\section{B. NON-ORTHOGONAL SECURE TRANSMISSION DESIGNS THROUGH AUXILIARY SIGNAL SUPERPOSITION}

1) NOMA with auxiliary signal superposition using time diversity $\left(C T_{3}\right.$

This technique proposes the design of a resilient, effective, and low complexity security scheme that can provide perfect secrecy, that is, zero information leakage to both internal and external eavesdroppers. The system does not require additional complexity to the IoT receiver. The receiver simply receives the signal and decodes it to get the intended information. The approach of this technique has some similarities with the previously proposed systems. The main difference is the use of auxiliary signals with time diversity, as opposed to pre-coding matrices. The auxiliary signals will be superimposed on user signals during the first and the second round of transmission as shown in Fig. 4 The auxiliary signals are specially designed matrices modeled from the channel characteristics of $u_{\text {ser }}$ and user $_{2}$ such that the signal received by an external eavesdropper is completely corrupted and internal eavesdropping is also prevented.

The superimposing of auxiliary signals is deliberately done to provide perfect secrecy against internal and external eavesdroppers as well as ensuring zero inter-user interference without using SIC at the legitimate users. Consider $\mathbf{x}_{1}$ and $\mathbf{x}_{2}$ as illustrated in the above sections. The transmitted signal from antenna- 1 during the first round is represented as:

$$
\mathbf{u}_{1}=\mathbf{x}_{1}+\mathbf{x}_{2}+\mathbf{r}_{1} .
$$

Similarly, the transmitted signal from antenna-2 during the second round is given as:

$$
\mathbf{u}_{2}=\mathbf{x}_{1}+\mathbf{x}_{2}+\mathbf{r}_{2} .
$$

The received signal at user $_{1}$ is the combined signal transmitted during the first and the second round through $\mathbf{H}_{11}$ and $\mathbf{H}_{12}$ depicted as:

$$
\hat{\mathbf{y}}_{1}=\mathbf{H}_{11}^{\mathrm{H}} \mathbf{y}_{11}+\mathbf{H}_{12}^{\mathrm{H}} \mathbf{y}_{12},
$$

where $\mathbf{y}_{11}$ is the received signal at user $_{1}$ during transmission round 1, and $\mathbf{y}_{12}$ is the received signal at user 1 


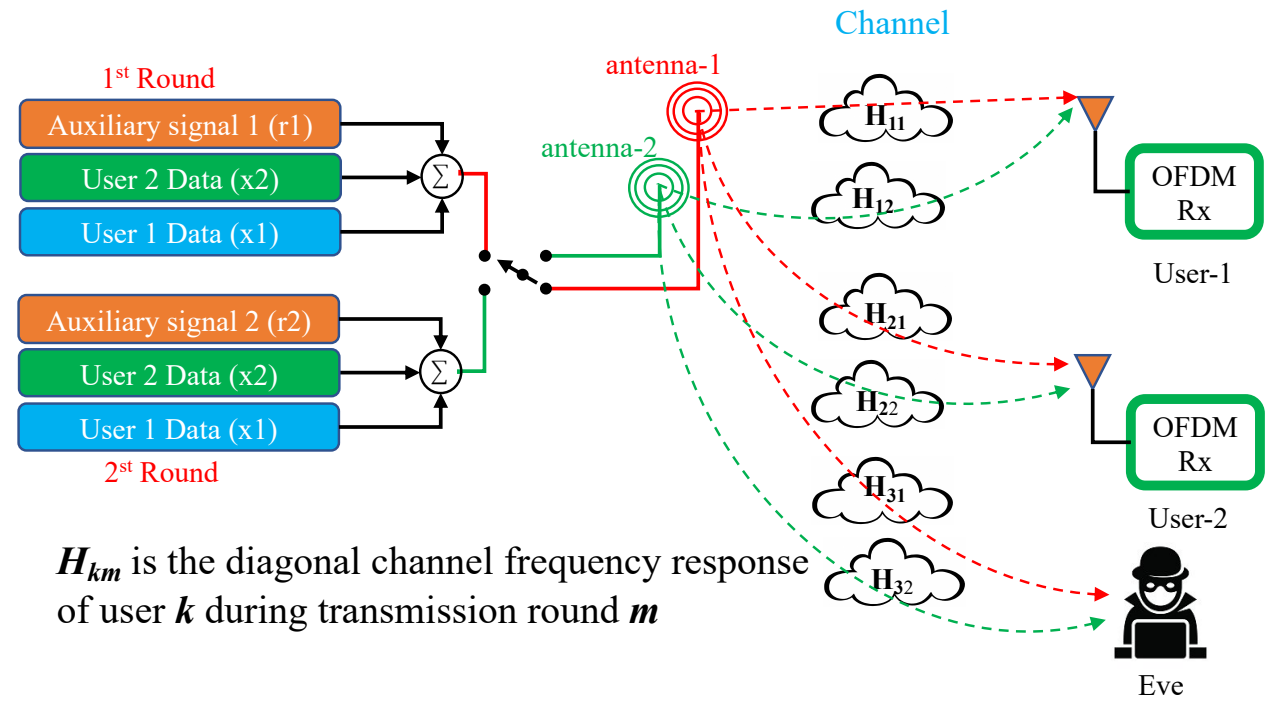

FIGURE 4. NOMA MISO OFDM detailed model. $\left(C T_{3}\right)$

during transmission round 2. Simplifying 28 further gives 29) and 30.

$$
\begin{gathered}
\hat{\mathbf{y}}_{1}=\left|\mathbf{H}_{11}\right|^{2} \mathbf{u}_{1}+\mathbf{H}_{11}^{\mathrm{H}} \mathbf{z}_{11} \\
+\left|\mathbf{H}_{12}\right|^{2} \mathbf{u}_{2}+\mathbf{H}_{12}^{\mathbf{H}} \mathbf{z}_{12}, \\
\hat{\mathbf{y}}_{\mathbf{1}}=\left(\left|\mathbf{H}_{11}\right|^{2}+\left|\mathbf{H}_{12}\right|^{2}\right) \mathbf{x}_{1}+\left(\left|\mathbf{H}_{11}\right|^{2}+\left|\mathbf{H}_{12}\right|^{2}\right) \mathbf{x}_{2} \\
+\left|\mathbf{H}_{11}\right|^{2} \mathbf{r}_{1}+\left|\mathbf{H}_{12}\right|^{2} \mathbf{r}_{2}+\mathbf{H}_{11}^{\mathbf{H}} \mathbf{z}_{11}+\mathbf{H}_{12}^{\mathrm{H}} \mathbf{z}_{12} .
\end{gathered}
$$

The received signal at $u_{s e r}$ is also the combined transmitted signal during round 1 and round 2 through $\mathbf{H}_{21}$ and $\mathbf{H}_{22}$ calculated as:

$$
\hat{\mathbf{y}}_{2}=\mathbf{H}_{21}^{\mathrm{H}} \mathbf{y}_{21}+\mathbf{H}_{22}^{\mathrm{H}} \mathbf{y}_{22},
$$

where $\mathbf{y}_{21}$ is the received signals at user $_{2}$ during round 1 through antenna- 1 and $\mathbf{y}_{22}$ is the received signal at user $_{2}$ during round 2 through antenna-2. Expanding (31) results in 32 and 33).

$$
\begin{gathered}
\hat{\mathbf{y}}_{\mathbf{2}}=\left|\mathbf{H}_{21}\right|^{2} \mathbf{u}_{1}+\mathbf{H}_{21}^{\mathbf{H}} \mathbf{z}_{21} \\
+\left|\mathbf{H}_{22}\right|^{2} \mathbf{u}_{2}+\mathbf{H}_{22}^{\mathbf{H}} \mathbf{z}_{22}, \\
\hat{\mathbf{y}}_{\mathbf{2}}=\left(\left|\mathbf{H}_{21}\right|^{2}+\left|\mathbf{H}_{22}\right|^{2}\right) \mathbf{x}_{1}+\left(\left|\mathbf{H}_{21}\right|^{2}+\left|\mathbf{H}_{22}\right|^{2}\right) \mathbf{x}_{2} \\
+\left|\mathbf{H}_{21}\right|^{2} \mathbf{r}_{1}+\left|\mathbf{H}_{22}\right|^{2} \mathbf{r}_{2}+\mathbf{H}_{21}^{\mathbf{H}} \mathbf{z}_{21}+\mathbf{H}_{22}^{\mathbf{H}} \mathbf{z}_{22},
\end{gathered}
$$

where $\mathbf{H}_{21}^{\mathbf{H}} \mathbf{z}_{21}$ and $\mathbf{H}_{22}^{\mathbf{H}} \mathbf{z}_{22}$ are noise elements. Similarly, the auxiliary signals superimposed in a time diversity system will make sure that the signal for each user is received without extra processing at the receiver and that the signals are secure from both internal and external eavesdropping.

Auxiliary signals $\mathbf{r}_{1}$ and $\mathbf{r}_{2}$ are designed as follows: As illustrated in 30 the first term is the desired term for user $_{1}$. Therefore, the auxiliary signals $\mathbf{r}_{1}$ and $\mathbf{r}_{2}$ will be designed such that the effect of the channels on $u s e r_{1}$ is removed as well as the interference by $u_{\text {ser }}$ on $u_{\text {ser }}$. Hence, the second, third, and fourth term in 30 should be equated to zero as shown below:

$$
\left(\left|\mathbf{H}_{11}\right|^{2}+\left|\mathbf{H}_{12}\right|^{2}\right) \mathbf{x}_{2}+\left|\mathbf{H}_{11}\right|^{2} \mathbf{r}_{\mathbf{1}}+\left|\mathbf{H}_{12}\right|^{2} \mathbf{r}_{2}=0 .
$$

Similarly, looking at 33, the second term of 33 is the desired term for user $_{2}$. Therefore, the auxiliary signals $\mathbf{r}_{1}$ and $\mathbf{r}_{2}$ will be designed such that the effect of the channels on user $_{2}$ is removed as well as the interference by user $_{1}$ on user $_{2}$. Hence, the first, third, and fourth term in 33 should be equal to zero and can be shown as:

$$
\left(\left|\mathbf{H}_{21}\right|^{2}+\left|\mathbf{H}_{22}\right|^{2}\right) \mathbf{x}_{\mathbf{1}}+\left|\mathbf{H}_{21}\right|^{2} \mathbf{r}_{\mathbf{1}}+\left|\mathbf{H}_{22}\right|^{2} \mathbf{r}_{\mathbf{2}}=0 .
$$

Equations (34) and (35) can jointly be solved to determine the values of auxiliary signals $\mathbf{r}_{1}$ and $\mathbf{r}_{2}$ as follows:

$$
\begin{gathered}
\mathbf{r}_{\mathbf{1}}=\frac{-\left(\left|\mathbf{H}_{21}\right|^{2}+\left|\mathbf{H}_{22}\right|^{2}\right) \mathbf{x}_{\mathbf{1}}-\left|\mathbf{H}_{22}\right|^{2} \mathbf{r}_{2}}{\left|\mathbf{H}_{21}\right|^{2}} \\
\mathbf{r}_{2}=\frac{\left(\left|\mathbf{H}_{11}\right|^{2}\left(\left|\mathbf{H}_{21}\right|^{2}+\left|\mathbf{H}_{22}\right|^{2}\right) \mathbf{x}_{\mathbf{1}}-\left|\mathbf{H}_{21}\right|^{2}\left(\left|\mathbf{H}_{11}\right|^{2}+\left|\mathbf{H}_{12}\right|^{2}\right) \mathbf{x}_{\mathbf{2}}\right.}{\left|\mathbf{H}_{12}\right|^{2}\left|\mathbf{H}_{21}\right|^{2}-\left|\mathbf{H}_{11}\right|^{2}\left|\mathbf{H}_{22}\right|^{2}},
\end{gathered}
$$

\section{2) NOMA with auxiliary signal superposition using spatial transmit diversity $\left(C T_{4}\right)$}

This design exploits NOMA with auxiliary signal superposition using spatial transmit diversity of base station for mMTC. The basic idea is to add channel-dependent auxiliary signals on top of the multiplexed users' (or IoT devices) signals, which are transmitted from two different antenna sources at the same time as shown in Fig. 5 The transmission is done such that the auxiliary signals completely cancel the interference only at the legitimate 
users, while causing severe degradation to the external eavesdropper.

Moreover, besides external security, the added signals are designed such that the inter-user interference between the legitimate users is completely removed.

The data for $u_{s e r_{1}}$ and $u s e r_{2}, \mathbf{x}_{1}$ and $\mathbf{x}_{2}$ respectively, is modulated using QAM modulation and the auxiliary signal specific for each antenna is added on top of the superimposed data signals as shown in Fig. 5. Thus, there is no need for using complex successive cancellation process at the receiver, resulting in reducing complexity, lowering power consumption, and decreasing latency. The transmitted signal from antenna- 1 after the addition of the auxiliary signal $\mathbf{r}_{\mathbf{1}}$ is given as follows:

$$
\mathbf{u}_{1}=\mathbf{x}_{1}+\mathbf{x}_{2}+\mathbf{r}_{1} \text {. }
$$

Similarly, the transmitted signal from antenna-2 after the addition of the auxiliary signal $\mathbf{r}_{2}$ is given as:

$$
\mathbf{u}_{2}=\mathbf{x}_{1}+\mathbf{x}_{2}+\mathbf{r}_{2} \text {. }
$$

The superimposed signals simultaneously sent from the two transmitters (i.e, A1 and A2) reach user $_{1}$ at the same time, hence, the received signal at user $_{1}$ is calculated as:

$$
\begin{gathered}
\hat{\mathbf{y}}_{1}=\mathbf{y}_{11}+\mathbf{y}_{12}=\mathbf{H}_{11} \mathbf{u}_{1}+\mathbf{H}_{12} \mathbf{u}_{2}+\mathbf{n}_{1} \\
\hat{\mathbf{y}}_{1}=\left(\mathbf{H}_{11}+\mathbf{H}_{12}\right) \mathbf{x}_{1}+\left(\mathbf{H}_{11}+\mathbf{H}_{12}\right) \mathbf{x}_{2}+\mathbf{H}_{11} \mathbf{r}_{1}+\mathbf{H}_{12} \mathbf{r}_{2}+\mathbf{n}_{1},
\end{gathered}
$$

where $\mathbf{y}_{11}$ is the received signal at user $_{1}$ from antenna-1, and $y_{12}$ is the received signal at user $_{1}$ from antenna-2 and $\mathbf{n}_{1}$ denotes the noise at user $_{1}$.

The received signal at $u e_{2}$ is also the combined transmitted signal from antenna- 1 and antenna- 2 calculated as:

$$
\begin{gathered}
\hat{\mathbf{y}}_{2}=\mathbf{y}_{21}+\mathbf{y}_{22}=\mathbf{H}_{21} \mathbf{u}_{1}+\mathbf{H}_{22} \mathbf{u}_{2}+\mathbf{n}_{2} \\
\hat{\mathbf{y}}_{2}=\left(\mathbf{H}_{21}+\mathbf{H}_{22}\right) \mathbf{x}_{1}+\left(\mathbf{H}_{21}+\mathbf{H}_{22}\right) \mathbf{x}_{2}+\mathbf{H}_{21} \mathbf{r}_{1}+\mathbf{H}_{22} \mathbf{r}_{2}+\mathbf{n}_{2},
\end{gathered}
$$

where $\mathbf{n}_{2}$ is the noise at user $_{2}$.

The values for the auxiliary signals $\mathbf{r}_{1}$ and $\mathbf{r}_{2}$, which completely cancel the interference between the users are found following the same reasoning detailed in the section above. As such, $\mathbf{r}_{1}$ and $\mathbf{r}_{2}$ are as follows:

$$
\begin{gathered}
\mathbf{r}_{1}=\mathbf{H}_{11}^{-1}\left(-\mathbf{x}_{2}-\mathbf{H}_{12} \mathbf{r}_{2}\right) \\
\mathbf{r}_{2}=\left(\mathbf{H}_{22}-\mathbf{H}_{21} \mathbf{H}_{11}^{-1} \mathbf{H}_{12}\right)^{-1}\left(-\mathbf{x}_{1}+\mathbf{H}_{21} \mathbf{H}_{11}^{-1} \mathbf{x}_{2}\right)
\end{gathered}
$$

Due to the particular design of the auxiliary signals $\mathbf{r}_{1}$ and $\mathbf{r}_{2}$, every user receives only their own data without the need for any SIC application and with complete security against internal and external eavesdroppers.

Note that calculating $r_{1}$ and $r_{2}$ can be done without too much complexity when the transmission system adopts the use of OFDM waveform due to the fact that all the channel matrices will be diagonal. Performing computations on a diagonal matrix such as finding the inverse consumes minimal resources compared to non diagonal matrices.

\section{PERFORMANCE ANALYSIS}

NOMA is regarded as a revolutionary technology for future wireless communication [31], specifically due to its ability to share resources among users. For instance, NOMA has been included in 3GPP LTE Release 13. Particularly, a downlink version of NOMA named MUST gained much attention amongst researchers and was adopted as a work item by 3GPP [31].

Since MUST was studied enough, we take it as a reference for the performance analysis of the proposed designs. Furthermore, the comparison between the proposed schemes and MUST will be done in terms of the following metrics:

1) Reliability.

2) Spectral efficiency (sum rate analysis).

3) Peak-to-average power ratio (PAPR).

4) Design complexity.

5) Security.

\section{A. SYSTEM PERFORMANCE}

In this section, a detailed comparison of the system performance is conducted. The performance metrics analysed include reliability, spectral efficiency through the sum rate analysis and peak to average power ratio.

\section{1) Reliability}

Reliability in communication networks is measured using many different indices as observed in the literature [32[33. In some cases, the reliability index is the terminal reliability, which is the probability that there exists at least one source-destination path $(x, y)$ in the network [b19, 32, 34, 33, 35, 36. This index assumes that the network has equal link capacity or that the link capacity is large enough to support a transmitted packet of any bandwidth. However, authors in 35 dispute this assumption citing that link capacity is a cost function and hence it is limited. Moreover, most communication systems have a given capacity requirement for transmitted data, hence, there exist a minimum bandwidth for the system to be successfully functional. In general, reliability is considered as the guarantee of messages transmitted from a transmitter to reach the intended user complete and uncorrected and in the order the message was sent.

Consider a two-user conventional NOMA system for instance, in a down-link power-domain NOMA(PD-NOMA known as MUST in 3GPP) paradigm, the two signals are allocated different powers depending on their location and channel condition and then transmitted. In contrast, the proposed designs use multiple antennas and rounds (as explained in section II) to send data for both users. The increased diversity in the proposed design significantly increases the reliability of the proposed system compared to the conventional NOMA systems. Moreover, the quality of the received signal at both users' terminals is highly dependent on the signal to noise ratio (SNR). The better the SNR value, the higher the reliability. For 


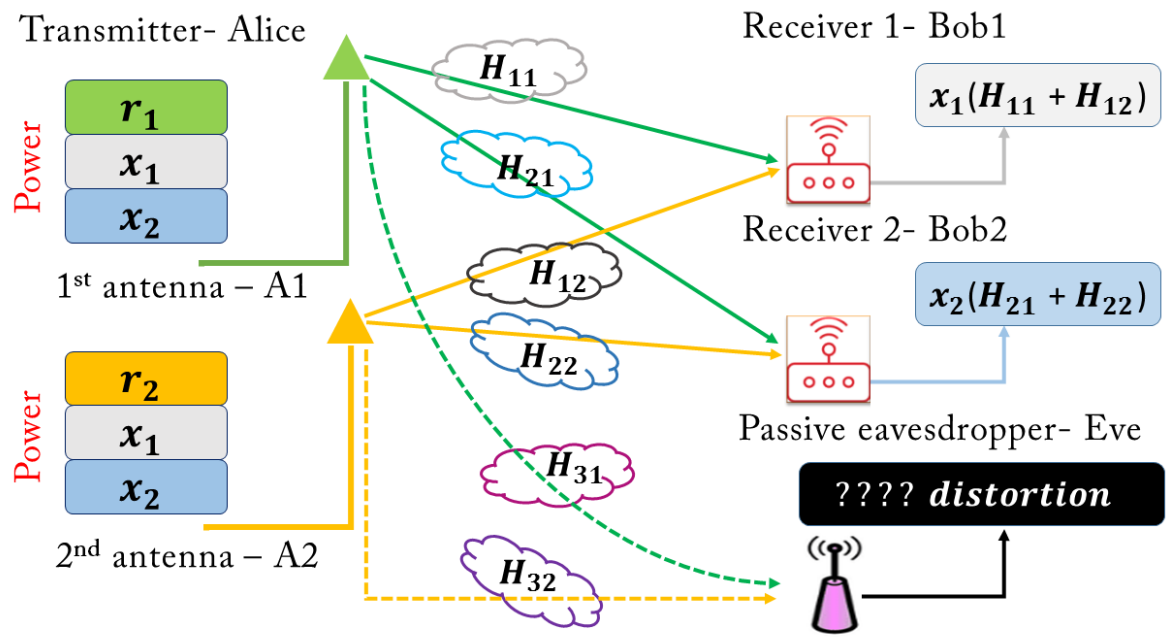

FIGURE 5. NOMA with auxiliary signal superposition using spatial transmit diversity $\left(C T_{4}\right)$

example, the SNR of OMA for near user (i.e, user $_{1}$ ) and far user (i.e, user $_{2}$ ) is:

$$
S N R_{\text {near }}=\frac{p_{\text {near }}}{n_{o}}, \quad S N R_{\text {far }}=\frac{p_{\text {far }}}{n_{o}},
$$

where, $p_{\text {near }}$ and $p_{\text {far }}$ indicates the allocated power for near and far users respectively, while $n_{o}$ is the channel noise. In contrast, the SNR for conventional NOMA for both near user and far user is given below:

$$
S N R_{\text {near }}=\frac{p_{\text {near }}}{n_{o}}, \quad S N R_{\text {far }}=\frac{p_{\text {far }}}{p_{\text {near }}+n_{o}},
$$

The signal decoded by the near user consists of only the channel noise, while that decoded by the far user has the channel noise as well as the near user signal (i.e. in NOMA, the far user treats the near user's signal as a noise). Looking at (47), It can be clearly observed that the SNR of individual users for a NOMA network is more degraded compared to OMA, and thus OMA is more reliable than NOMA.

Unlike NOMA where a combination of a far and a near user is required, the proposed paradigms discussed in section II can also work in power-balanced scenarios and thus achieve a reliability performance similar to that of an OMA system. Moreover, although in the case of power imbalance between the users the design is similar to that of conventional NOMA, the design complexity of the proposed schemes is much less due to the elimination of the SIC technique.

Bit error rate (BER) is also a common measure of reliability in many communication systems. It measures the probability that a bit sent from a transmitter is received without any alterations at the receiver. In this work, the BER for each of the four proposed novel NOMA schemes is computed. Analytical data fitting method proposed in
[37] is used to compute the theoretical BER of the proposed NOMA schemes. The statistics of the instantaneous signal-to-noise ratio (SNR), $\gamma_{b}$ is first calculated for the legitimate user utilizing each scheme. The probability density function for the effective instantaneous SNR is calculated as [37:

$$
P_{\gamma b}\left(\gamma_{b}\right) \approx\left(\frac{1}{\omega}\right)^{\mu} \frac{1}{\Gamma(\mu)} \frac{\Omega^{\frac{3}{2}} \sqrt{\gamma_{b}}}{\hat{\gamma}_{b}^{\frac{3}{2}}} \exp \left(-\frac{1}{\omega} \frac{\omega \gamma_{b}}{\hat{\gamma}_{b}}\right),
$$

where $\Omega, \hat{\gamma}_{b}$, and $\Gamma(\mu)$ are the mean square of the sub-channels, average SNR, and the gamma function respectively. $P_{\gamma b}\left(\gamma_{b}\right)$ can then be used to calculate the BER.

$$
B E R_{b}=\frac{1}{2} \int_{0}^{\infty} \operatorname{erfc}\left(\sqrt{\gamma_{b}}\right) P_{\gamma_{b}}\left(\gamma_{b}\right) d \gamma_{b}
$$

Substituting $P_{\gamma b}\left(\gamma_{b}\right)$ results to:

$$
\begin{array}{r}
B E R_{b}=\frac{1}{2} \int_{0}^{\infty} \operatorname{erfc}\left(\sqrt{\gamma_{b}}\right)\left(\frac{1}{\omega}\right)^{\mu} \frac{1}{\Gamma(\mu)} \\
\frac{\Omega^{\frac{3}{2}} \sqrt{\gamma_{b}}}{\hat{\gamma}_{b}^{\frac{3}{2}}} \exp \left(-\frac{1}{\omega} \frac{\omega \gamma_{b}}{\hat{\gamma}_{b}}\right) d \gamma_{b} .
\end{array}
$$

Simplifying [50 results in 51] 37.

$$
\begin{gathered}
B E R_{b} \approx \frac{\left(\frac{1}{\omega}\right)^{\mu} \frac{1}{\Gamma(\mu)} \frac{\Omega^{\frac{3}{2}}}{\hat{\gamma}_{b}^{\frac{3}{2}}}}{2 \sqrt{\pi}} \\
\left(\frac{\arctan \left(\sqrt{\frac{1}{\omega} \frac{\omega}{\hat{\gamma}_{b}}}\right)}{2 \frac{1}{\omega}{\frac{\omega}{\hat{\gamma}_{b}}}^{3 / 2}}-\frac{1}{2 \frac{1}{\omega} \frac{\omega}{\hat{\gamma_{b}}}\left(1+\frac{1}{\omega} \frac{\Omega}{\hat{\gamma}}\right)}\right)
\end{gathered}
$$

where, $\arctan (\cdot)$ is the inverse tangent. 
Fig. 6 indicate the BER performance of the proposed schemes compared to conventional NOMA. Theo. Near user and Theo. Far user are the theoretical BER for near and far users utilizing conventional NOMA. The far user has the worst channel as well as more power allocation. The figure indicates that $C T_{2}$ has the best BER performance, followed by $C T_{1}$ then $C T_{3}$ then conventional NOMA far user and $\mathrm{CT}_{4}$ and finally conventional NOMA near user. Conventional NOMA far user and $C T_{4}$ have very similar BER performance, where up to an SNR of $10 \mathrm{~dB}$ conventional NOMA far user's BER performance is slightly better than $C T_{4}$, and after $10 \mathrm{~dB} C T_{4}$ performs slightly better.

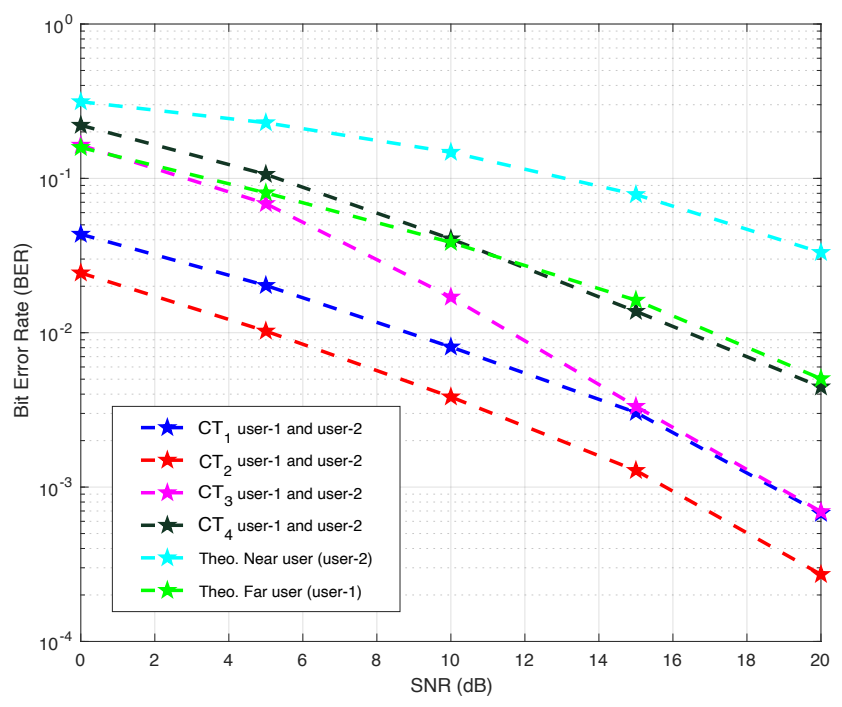

FIGURE 6. BER performance of NOMA-inspired proposed designs and conventional NOMA. For all the proposed designs user-1 and user-2 have the same BER performance)

\section{2) Sum Rate Analysis for the proposed designs}

This section presents a comparative analysis between the four designs (i.e, $C T_{1}, C T_{2}, C T_{3}, C T_{4}$ ) and the conventional PD-NOMA (MUST) in terms of the achievable users' data rates. Fig. 7 is a comparative plot including a common representation of the signal transmission/reception in all the four proposed designs along with the conventional NOMA system model for 2 users.

Regardless of the transmission mechanism employed (i.e, round or simultaneous signal transmission), the received signal at $u s e r_{i}, i=\{1,2\}$ denoted by $y_{i}$ is the summation of three terms including a desired term for the user $_{i}$, an undesired term (i.e, interference term) and a noise component as shown in the equation below:

$$
y_{i}=S_{i, j}+I_{i, j}+n_{i, j}, i=1,2
$$

where, $S_{i, j}$ and $n_{i, j}$ stand for the desired term and the noise term for $u s e r_{i}$, respectively. The index $j$ refers to the technique used (i.e, $C T_{1}, C T_{2}, C T_{3}, C T_{4}$ ). Note that, the interference term (i.e, $I_{i, j}$ ) in 52 is the interference caused by $u$ ser $_{i}$ on another user. In the case of two users, the term $I_{1,2}$ in the equation of $u_{s e r}$ (i.e, $y_{1}$ ) is the interference caused by user 1 on user $_{2}$. Similarly, the term $I_{2,1}$ in the equation of user $_{2}$ (i.e, $y_{2}$ ) is the interference caused by user $_{2}$ on user $_{1}$.

The comparison is performed by quantifying the sum rate of each scheme. The achievable rate for user $_{i}$ employing design $C T_{j}, j=1,2,3,4$ is denoted by $R_{i, j}$ and is defined as:

$$
R_{i, j}=\log _{2}\left(1+S I N R_{i, j}\right),
$$

where $S I N R_{i, j}$ denotes the signal-to-interference plus noise ratio for user $_{i}$ using design $C T_{j}$. The overall sum rate for any scheme $C T_{j}$ is defined as the summation of the rates of all the users employing this scheme. In our analysis, we consider a downlink setup for 2 users. That's the sum rate of the proposed design $C T_{j}$ is defined by:

$$
R_{D_{j}}=\log _{2}\left(1+S I N R_{u s e r_{1}, j}\right)+\log _{2}\left(1+S I N R_{u s e r_{2}, j}\right),
$$

or,

$$
R_{D_{j}}=\log _{2}\left[\left(1+S I N R_{\text {user }_{1}, j}\right) \times\left(1+\operatorname{SINR}_{\text {user }_{2}, j}\right)\right] .
$$

Moreover, as the received signal for $u_{s e r}$ employing design $C T_{j}, j=1,2,3,4$ follows the format expressed by the relation in [52, $S I N R_{i, j}$ is by definition:

$$
\operatorname{SINR}_{i, j}=\frac{S_{i, j}}{I_{i, j}+\sigma_{n_{i, j}}^{2}},
$$

where, $\sigma_{n_{i, j}}^{2}$ denotes the variance of the noise term.

In Fig. 7. the lower part of the figure depicts a downlink power domain NOMA setup for two users. It is assumed that user $_{2}$ is the closest to the base station and is assumed to have a better channel condition than user $_{1}$ (i.e, $h_{1}^{2} \leq h_{2}^{2}$ ). The total base station transmit power is denoted with $P_{s}$ and the shares of the power between user $_{1}$ and user $_{2}$ are $a_{1} \times P_{s}$ and $a_{2} \times P_{s}$, respectively, where $a_{2} \leq a_{1}$ and $a_{1}+a_{2}=1$ as in conventional NOMA users are allocated power based on their channel conditions.

The transmitted superimposed signal $s$ can be written:

$$
s=\left(\sqrt{a_{1} \times P_{s}}\right) x_{1}+\left(\sqrt{a_{2} \times P_{s}}\right) x_{2},
$$

where $x_{1}, x_{2}$ respectively denote the data for $u_{s e r_{1}}$ and user $_{2}$ with unit energy.

Moreover, the received signal at $u$ ser $_{1}$ and $u_{\text {ser }}$ can be expressed as:

$$
y_{1}=h_{1} s+n_{1},
$$

$$
y_{2}=h_{2} s+n_{2},
$$



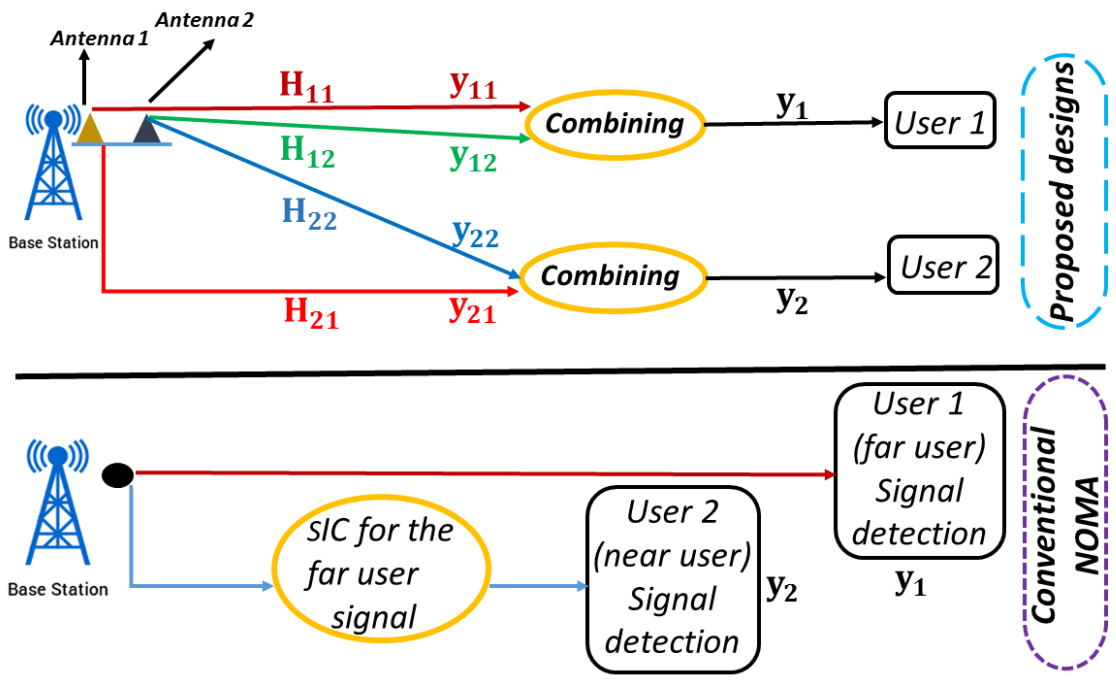

FIGURE 7. A common representation for the system model of the four schemes (i.e, $C T_{1}, C T_{2}, C T_{3}, C T_{4}$ ) along with the system of conventional NOMA for two users. Conventional NOMA is designed without taking into account that we have always redundant transmission to protect the transmitted data against channel impairment.

where $n_{1}, n_{2}$ are complex additive Gaussian noise values with zero-mean and real variance, for user $_{1}$ and $u s e r_{2}$ respectively. That is, $n_{1}=N\left(0, \sigma_{1}^{2}\right), n_{2}=N\left(0, \sigma_{2}^{2}\right)$.

In this setup, user $_{2}$ employs SIC to detect its own signal by first detecting the signal of user $_{1}$ which has more power than its own signal. The signal of user $_{1}$ is then subtracted from the overall received signal at user $_{2}$ (i.e, $y_{2}$ ) to obtain the signal for user $_{2}$.

The signal-to-interference plus noise ratio derivations for downlink NOMA are detailed in [4] and can be written, for user $_{1}$, as:

$$
S I N R_{1}=\frac{a_{1} P_{s} h_{1}^{2}}{a_{2} P_{s} h_{1}^{2}+\sigma_{1}^{2}}
$$

and for user $_{2}$ as:

$$
S I N R_{2}=\frac{a_{2} P_{s} h_{2}^{2}}{\sigma_{2}^{2}} .
$$

Furthermore, the achievable rate for downlink NOMA can be found by summing the individual user rates as follows:

$$
R_{N O M A}=\log _{2}\left(1+S I N R_{1}\right)+\log _{2}\left(1+S I N R_{2}\right),
$$

or

$$
R_{N O M A}=\log _{2}\left[\left(1+S I N R_{1}\right) \times\left(1+S I N R_{2}\right)\right] .
$$

\section{3) Peak to average power ratio (PAPR)}

The general definition of PAPR of an OFDM signal $x(t)$ is the ratio between the maximum instantaneous power and the average power $[38$.

$$
\operatorname{PAPR}[x(t)]=\frac{\max _{0 \leq t \leq N T}\left[|x(t)|^{2}\right]}{P_{a v}},
$$

where $P_{a v}$ is the average power of $x(t)$. High PAPR results in performance degradation in a communication system and it is one of the most challenging issue in OFDM systems [38. Various PAPR reduction techniques have been proposed in the literature [38, 39], the techniques can generally be categorized into two categories, namely signal scrambling and signal distortion techniques. Some of the proposed methods include: clipping and filtering [40], Coding Schemes [41, 42, 43, 44], partial transmission sequence (PTS) and selective mapping (SLM) 45, 46 47], phase optimization [48, 49], nonlinear companding transforms [50, 51, 52, tone reservation (TR) and tone injection (TI) [53], constellation shaping [54 55, 56], and pre-scrambles [57].

The PAPR of a communication technique is therefore critical in determining the performance of the system [58]. In this section, we compare the PAPR of the proposed NOMA-inspired designs to conventional NOMA. Fig. 8 depicts the PAPR comparison for a two transmitter system for proposed schemes and conventional NOMA. As it can be observed, most of the proposed schemes have better PAPR performance than conventional NOMA. Moreover, the proposed NOMA designs work in a power balanced scenarios and do not require the intentional pairing of near and far users as is the case of conventional NOMA.

\section{B. COMPLEXITY ANALYSIS LATENCY}

The comparative diagram of Fig. 7 along with the individual transceivers for the proposed designs (i.e, Fig. 2 234 45 infer the simplicity of the design of these schemes compared to conventional NOMA.

In particular, none of the proposed designs employ the SIC principle, which is the main source of complexity and latency in conventional NOMA. On the other hand, the proposed schemes completely cancel the interference 


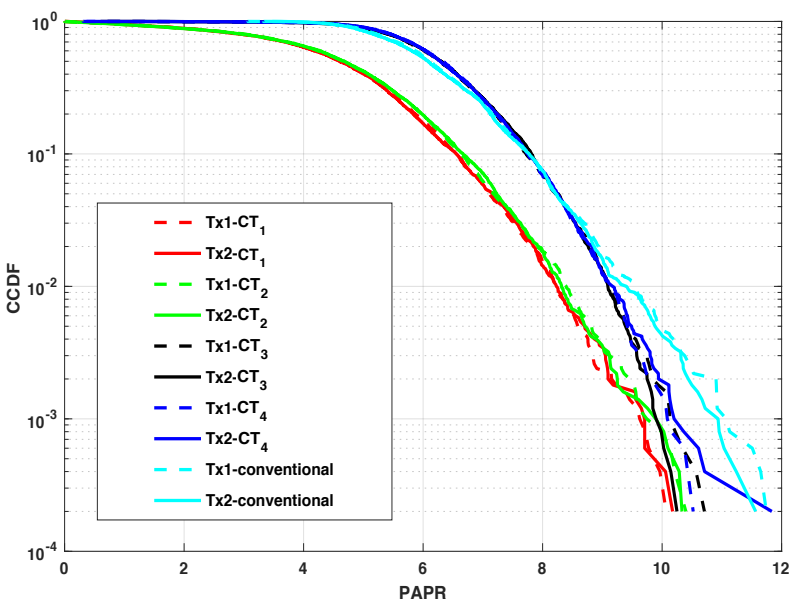

FIGURE 8. Peak to average power ratio (PAPR)

between the multiplexed users using signal precoding and auxiliary signal addition. The required processing such as signal precoding or signal addition is performed only at the base station, freeing the receiver from complex processing operations. As such, the proposed designs are suitable for IoT devices with limited processing capabilities due to their small sizes and low battery lives.

\section{SECURITY}

In this section, we will briefly discuss the security concept in the proposed paradigms compared to conventional NOMA. The security part of the proposed paradigms will be broadly analyzed in future works dedicated to each technique including [59] for $\left(C T_{2}\right)$ and $\left[60\right.$ for $\left(C T_{3}\right)$, however, this section is intended to introduce the novelty of the security concept in the proposed techniques. NOMA's edge in comparison to other communication techniques is the ability of multiple users to use the same resources. Nevertheless, this advantage also comes with high security risks, where an eavesdropper (internal or external) might be able to intercept messages of users utilizing the same resource [61]. Therefore, NOMA is highly susceptible to internal eavesdropping in the case of an untrusted user (due to the decoding of the far user's signal by the near user (i,e. SIC)) as well as external eavesdropping.

According to 61, physical layer security (PLS) has emerged as a promising security technique that may replace conventional security methods such as cryptography in future wireless communication and address the security concerns faced by future IoT applications 62 63. Cryptography based methods are the main security techniques used by conventional NOMA and other wireless communication technologies. However, cryptographic methods have multiple weaknesses that are not suitable for future wireless communication systems [37]. Such drawbacks include: key sharing and management problems, where the decentralized and homogeneous na- ture of future wireless networks will make it very difficult for key sharing and management. Also, high processing power used in cryptography [37], is not friendly for lowpower future wireless devices. Finally, cryptography lacks the flexibility to provide different levels of security for different services and scenarios.

The proposed NOMA schemes will utilize PLS to provide a secure communication channel against internal and external eavesdroppers. Two novel methods are used to design the security schemes, these methods are very different to what is found in literature. In the first case, we use pre-coding matrices as described in section II A. In this case, channel dependent pre-coding matrices are calculated and used as shown in (1) and 2) to provide perfect secrecy against internal and external eavesdroppers without any processing at the receiver. In the second case, channel depended auxiliary signals are calculated and added to the user signals as shown in 26) and 27). Similarly, the auxiliary signals added will provide perfect secrecy against internal and external eavesdroppers without additional signal processing at the receiver.

\section{CONCLUSION}

This work has proposed four novel NOMA-inspired communication schemes for enhancing security and reliability of low-complexity massive machine type communications. The proposed designs have out-performed conventional NOMA in performance metrics such as security, complexity, reliability and others. In the future, we intent to investigate and design paradigms for more than two users. Moreover, each of the proposed paradigms in this preliminary, trailer paper is going to be extensively studied and deeply investigated in terms of mathematical model, system design and performance analysis in separate future extensions of this work.

\section{ACKNOWLEDGEMENTS}

We would like to acknowledge and thank Prof. Theodoros Tsiftsis for his feedback on this work, where he recommended and suggested valuable changes that helped improve the quality of the paper. 


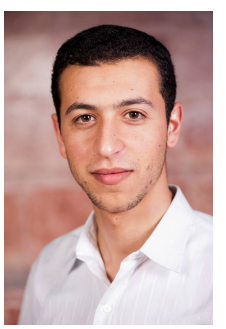

JEHAD M. HAMAMREH is currently an Assistant Professor with the Electrical and Electronics Engineering Department, Antalya International (Bilim) University, Turkey. He received the Ph.D. degree in electrical-electronics engineering and cyber systems from Istanbul Medipol University, Turkey, in 2018. He worked as a Researcher at the Department of Electrical and Computer Engineering in Texas AM University. He is the inventor of $8+$ Patents, and He has authored more than 55+ peer reviewed scientific papers along with several book chapters. His innovative patented works won the golden, silver and bronze medals by numerous international invention contests and fairs. His current research interests include wireless physical and MAC layers security, orthogonal frequency-division multiplexing multiple-input multiple-output systems, advanced waveforms design, multidimensional modulation techniques, and orthogonal/nonorthogonal multiple access schemes for future wireless systems. He is a regular investigator and referee for various scientific journals as well as a TPC member for several international conferences. He is an Editor at RS-OJICT and Frontiers in Communications and Networks.

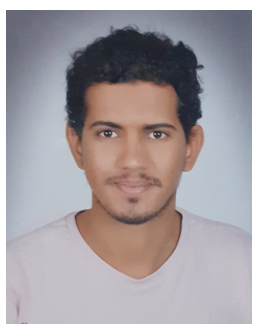

MOHAMEDOU ABEWA received the B.Sc.degree in electrical and electronics engineering from Antalya International (Bilim) University, Turkey in 2019. He is currently pursuing a masters degree in electrical and computer engineering at the same university.

His current research interests include waveform design and particularly non-coherent wireless designs, Multiple Access schemes, and physical layer security for wireless systems.

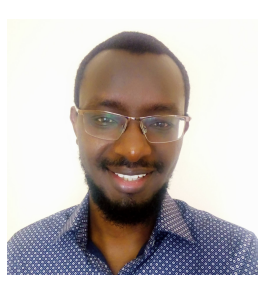

JOEL P. LEMAYIAN received the B.Sc. degree in electrical and electronics engineering from Middle East Technical University Turkey, in 2017. He is presently pursuing the master's (M.Sc.) degree in electrical and computer engineering. He is currently with Antalya Bilim University, Turkey.

He has worked as a research assistant in both Middle East Technical University and Antalya Bilim University in IoT lab and Neuroscience and wireless communications lab respectively. He is an author of numerous journals, conference papers, and book chapters. His research interests include UAVs, Physical Layer Security (PLS), 6G Communication networks, Artificial Intelligence, Machine Learning, and the Internet of Things (IoT) applications. 